"Submitted to the 1995 Particle Accelerator Conference, Dallas, Texas, May 1-5, 1995.

\title{
FIRST RESULTS with a NONLINEAR DIGITAL ORBIT FEEDBACK SYSTEM at the NSLS *
}

\author{
Eva Bozoki, Aharon Friedman and Susila Ramamoorthy \\ National Synchrotron Light Source, Brookhaven National Laboratory, Upton, NY 11973
}

Abstract

We report on the first experimental results with a nonlinear digital orbit feedback system for the NSLS X-ray ring. The system uses the existing RF receivers and orbit corrector magnets (trims) as well as parts of the NSLS control components. The orbit measurement micro was upgraded to a Motorola $68040 \mathrm{CPU}$ in order to achieve the necessary data rate. Filtering and orbit correction calculations are done in a dedicated HP $742 \mathrm{rt}$ micro. The system operates at a $555 \mathrm{~Hz}$ data rate, and achieves a bandwidth of $15-20 \mathrm{~Hz}$.

\section{INTRODUCTION}

Orbit stability is an important issue for storage rings, like the ones at the NSLS. Brightness of the photon source and beam lifetime can deteriorate due to even small motions in the beam orbit. The orbit can be stabilized with feedback systems. Digital feedback systems Ref. [1] are very flexible, since the filter and orbit correction algorithms are programmed and any change does not require changes in the hardware. This is a clear advantage over the presently used analog hardware based feedback systems.

\section{ORBIT CORRECTION ALGORITHM}

In implementing the digital feedback system, we are using the eigenvector decomposition based orbit correction method described in Refs. [2], [3]. This method yields the 'minimum' kick vector required for a desired accuracy of orbit correction.

\section{FILTERING}

The feedback system and its elements are illustrated in Fig. 1.

$G$ represents the effect of the vacuum chamber. It behaves like a single pole low pass filter, with the pole at $\approx 25$ Hz: ${ }^{1}$

$$
G(s)=\frac{2 \pi 25}{s+2 \pi 25}
$$

$H_{A A}$ is an "anti aliasing" filter which limits the bandwidth of the signal in order to prevent aliasing (folding) of the signal spectrum after the $D / A$ conversion. It is a low pass filter with a single pole at $\approx 80 \mathrm{~Hz}$ :

$$
H_{a a}(z)=\frac{2 \pi 80}{s+2 \pi 80}
$$

\footnotetext{
-Work performed under the auspices of the U.S. Dept. of Energy under contract no. DE-AC02-76CHooo16.

${ }^{1}$ In this section $\mathrm{G}(\mathrm{s})$ is the Laplace-transform of an $\mathrm{g}(\mathrm{t})$ continuous signal $(s=j 2 \pi f)$, and $H(z)$ is the Z-transform of an $h(n)$ discrete signal $\left(z=\exp \left(j 2 \pi f t_{s}\right)\right)$, where $t_{s}$ is the sampling time.
}

$H_{d}(z)=z^{-3}$ represents the phase delay due to sampling time, computation time and conversion time.

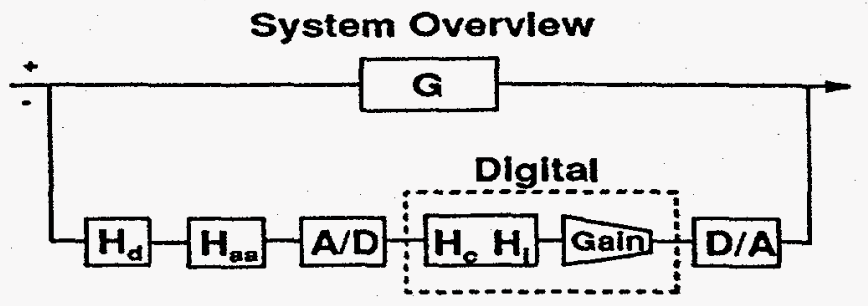

Figure. 1. Block diagram of the ring and feedback loop showing the elements of the feedback system.

The $H_{c}$ filter is designed to compensate for elements in the system $G$ that may be limiting the bandwidth and adding phase retardation to the system. This filter is a high pass filter:

$$
H_{c}(z)=\frac{1}{G(z)}=8.026 \frac{1-0.751 z^{-1}}{1+z^{-1}}
$$

$H_{I}$ is an integrator, which together with the gain is used to limit the bandwidth of the system and to stabilize it.

$$
H_{i}(z)=1.69 \times 10^{-3} \frac{1+z^{-1}}{1-z^{-1}}
$$

Note that a pure integrator generates infinite gain at DC. Hence, the correction at DC is absolute.

The closed loop response of this system is

$$
T=\frac{G}{1+g_{o} G H_{\text {tot }}}, \quad \text { where } H_{\text {tot }}=H_{a a} H_{d} H_{c} H_{i} \text {, }
$$

$\mathrm{T}$ is plotted as a function of the $\mathrm{f}$ frequency on Fig. 2

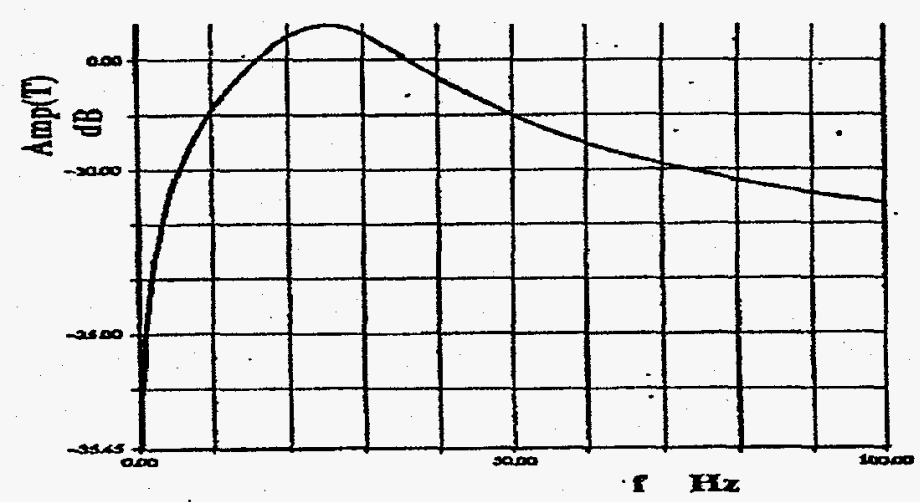

Figure. 2. Calculated amplitude response. 


\section{HARDWARE}

The feedback system consists of three micros; the HP742rt CPU based feedback micro, the Motorola-167 CPU based orbit micro and the Motorola-133 CPU based trim micro. The communication between the micros is done by the Bit-3 bus adapter boards through shared memory in the trim and in the orbit micro. The basic layout is shown in Fig. 3.

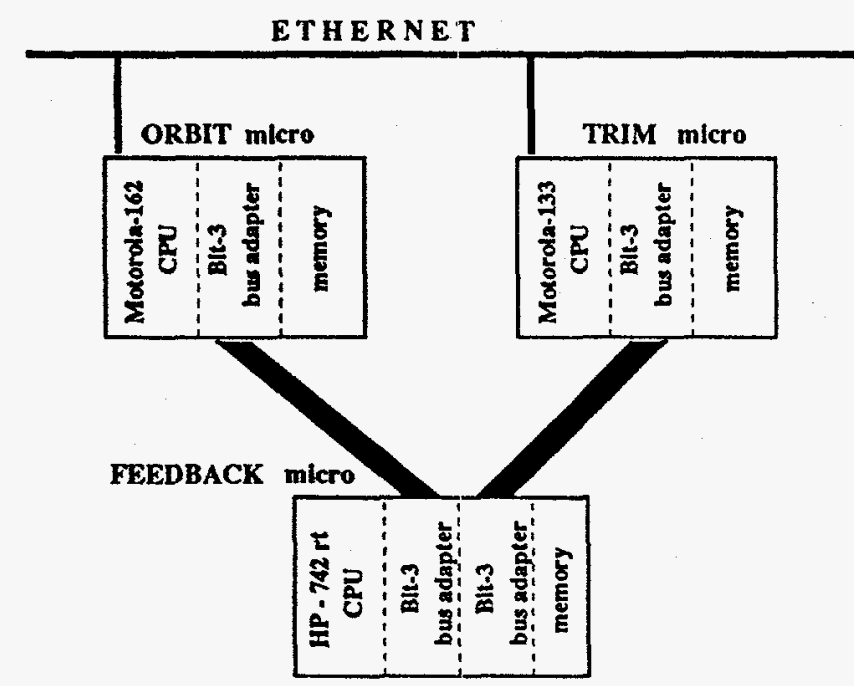

Figure. 3. Layout of the feedback system.

The orbit micro samples the PUE data at $555 \mathrm{~Hz}$ rate and the data is stored in memory. This memory is mapped by the adapter board to the address space of the feedback micro which calculates the orbit correction and optimizes it. The data communication between the feedback and trim micros is again through memory mapping. The trim micro then controls the power supplies of the orbit corrector magnets. The most computational intensive task is that of the feedback micro. Hence, we chose an HP 742rt, which we estimate to run six times faster than a Motorola 167/162 for this kind of application.

Though the orbit is sampled at $555 \mathrm{~Hz}$ rate for the feedback, the data at $32 \mathrm{~Hz}$ rate is available for the workstations for existing control programs [4] such as Real Time Orbit, Fast Orbit History, etc. Thus the PUE and feedback micros are isolated from the general control network.

To prevent aliasing problem the PUEs are set up with an analog low pass filter.

If it becomes necessary, we will include a control micro in the design to isolate the feedback system from the general control network. The need may arises, since the micros are expected to operate at close to full load and any requests addressed to them on the network may slow them down, reducing the feedback rate.

\section{SOFTWARE}

The orbit and trim micros use the existing NSLS real time monitor [5]. The programming of the monitor, as well as of the orbit and trim micros were modified such that the feedback micro can place the read points and set point into the shared memory, and that the data collection is synchronized with the feedback micro. The PUE readpoints are sampled with $555 \mathrm{~Hz}$ for the feedback micro but updated with $32 \mathrm{~Hz}$ for general use. A new monitor was written for the feedback micro, based on the HP-RT operating system. The orbit correction code is a modification of the code that was used for orbit correction in Refs. [2], [3]. This is an object oriented code written in $\mathrm{C}++$.

\section{FIRST RESULTS}

The feedback system was run successfully on the vertical plane of the NSLS X-ray ring, using all 40 orbit correctors and 48 PUEs. The frequency response of the closed system was measured and compared with the frequency response without feedback. One of the orbit correctors was driven by the output of an HP Dynamic Signal Analyzer in the $.1-100 \mathrm{~Hz}$ frequency region and the amplitude and phase response of one of the PUE's was recorded. The results are shown on Fig. 4.

FREQ RES:
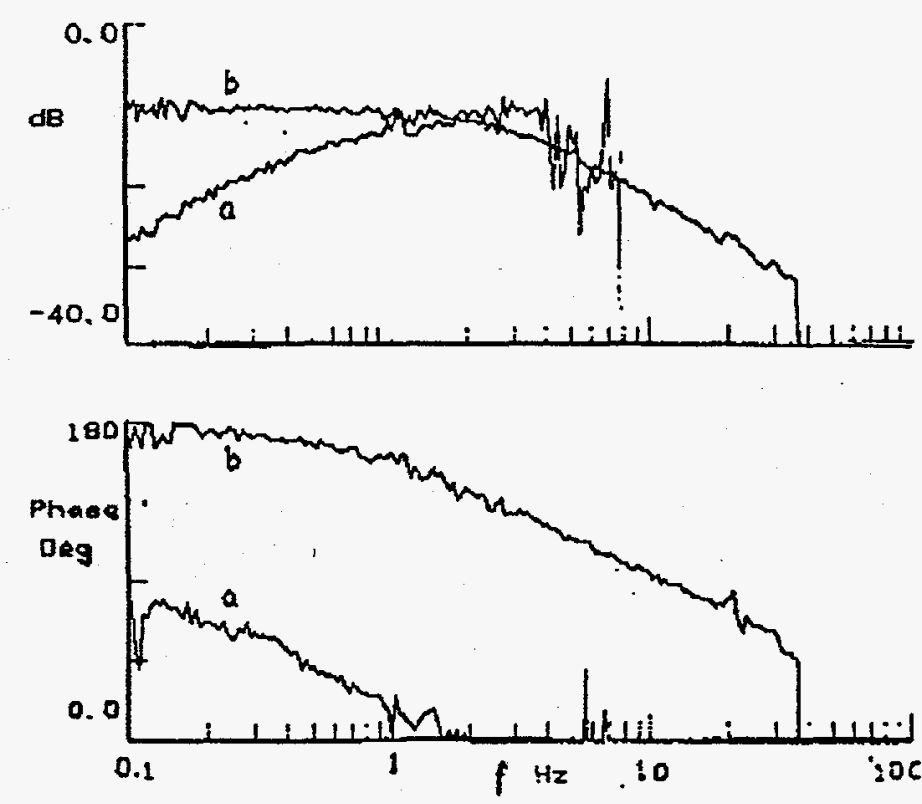

Figure. 4. Measured amplitude and phase response.

The DC noise reduction is $\approx 40 \mathrm{db}$ and the effective bandwidth is $15-20 \mathrm{~Hz}$ with a small noise amplification between $20-30 \mathrm{~Hz}$. These results are in good agreement with the calculated closed loop response shown in Fig. 2.

\section{FUTURE PLANS}

In order to achieve a higher $(1.5-2.0 \mathrm{kHz})$ orbit sampling rate, we will replace the present orbit reading system with Analogic 16-bit, $400 \mathrm{kHz}$ data acquisition boards and 32 channel multiplexers. 


\section{DISCLAIMER}

Portions of this document may be illegible in electronic image products. Images are produced from the best available original document. 
We are planning to add a notch filter at $60 \mathrm{~Hz}$ in order to effectively suppress a sometimes strong noise at that frequency. In this case the $H_{t o t}$ in Eq. (5) is replaced by

$$
H_{t o t}=H_{a a} H_{d} H_{c}\left(H_{i}+g_{n} H_{n}\right) \text {. }
$$

The calculated amplitude response of the total closed system with the wide band low pass filter and the notch filter is shown in Fig. 5.

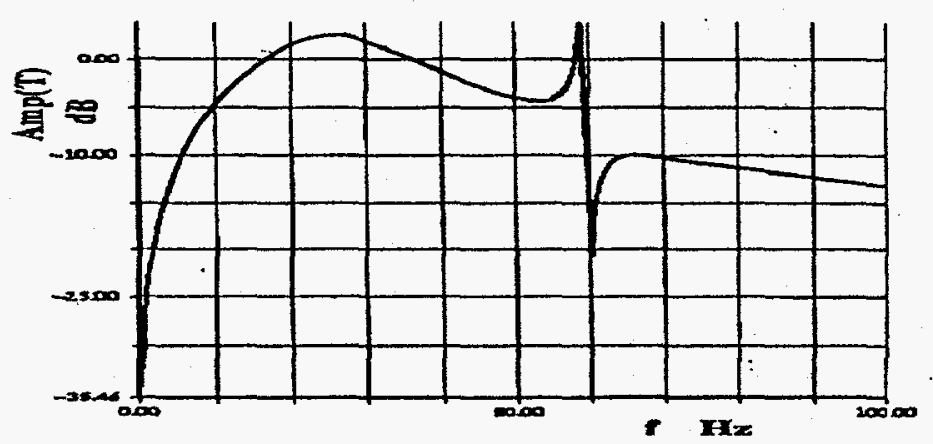

Figure. 5. Calculated amplitude response with notch filter.

\section{ACKNOWLEDGEMENT}

The auathors are grateful to the team of technicians lead by Wayne Rambo for their help.

\section{References}

[1] A. Friedman and E. Bozoki, "A Digital Feedback System for Transverse Orbit Stabilization," Proc. EPAC, p.1586, 1994.

[2] E. Bozoki and A. Friedman, "Optimization Method for Orbit Correction in Accelerators," Proc. IEEE PAC, p.105, 1993.

[3] A. Friedman and E. Bozoki, "Use of Eigen Vectors in Understanding and Correcting Storage Ring Orbits," NIM A, vol. 344, pp. 269-277, 1994.

[4] J. Smith, S. Ramamoothy, Y. Tang, J. Flannigan, S. Sathe, J. Kean, S. Krinsky, "NSLS Control System Upgrade Status", Proc. IEEE PAC, p.1952, 1993.

[5] S. Ramamoothy and J. Smith, "NSLS Control Monitor and its Upgrade", Proc. IEEE PAC, p.1849, 1993.

\section{DISCLAIMER}

This report was prepared as an account of work sponsored by an agency of the United States Government. Neither the United States Government nor any agency thereof, nor any of their employees, makes any warranty, express or implied, or assumes any legal liability or responsibility for the accuracy, completeness, or usefulness of any information, apparatus, product, or process disclosed, or represents that its use would not infringe privately owned rights. Reference herein to any specific commercial product, process, or service by trade name, trademark, manufacturer, or otherwise does not necessarily constitute or imply its endorsement, recommendation, or favoring by the United States Government or any agency thereof. The views and opinions of authors expressed herein do not necessarily state or reflect those of the United States Government or any agency thereof. 